\title{
Effects of Ethyl Pyruvate on Bile Duct Ligation-Induced Liver Fibrosis by Regulating Nrf2 Pathway and Proinflammatory Cytokines in Rats
}

\author{
Yonghua Zong, ${ }^{1,2}$ Mingxiao Zhang $\mathbb{D}^{1},{ }^{1}$ Shuai $\mathrm{Li}^{3}{ }^{3}$ Wenqian Qi, ${ }^{4} \mathrm{Juan} \mathrm{Li}^{3}{ }^{3}$ Tonghua Liu, ${ }^{2}$ \\ Huijun Yang, ${ }^{3}$ Chen Lu $\mathbb{D},{ }^{5}$ and Xiaosong $\mathrm{Hu} \mathbb{( i )}^{3}$ \\ ${ }^{1}$ Luoyang Polytechnic, Luoyang 471000, China \\ ${ }^{2}$ Tibet Traditional Medicine University, Lhasa 850000, China \\ ${ }^{3}$ Chengdu Medical College, Sichuan 610500, China \\ ${ }^{4}$ Qingdao Binhai University, Qingdao 266555, China \\ ${ }^{5}$ University of Electronic Science and Technology of China, Sichuan 610500, China
}

Correspondence should be addressed to Mingxiao Zhang; 595112042@qq.com, Chen Lu; 1025032768@qq.com, and Xiaosong Hu; woodwind@sohu.com

Yonghua Zong and Mingxiao Zhang contributed equally to this work.

Received 24 February 2019; Revised 2 May 2019; Accepted 19 May 2019; Published 20 December 2019

Academic Editor: Mario Pirisi

Copyright ( 2019 Yonghua Zong et al. This is an open access article distributed under the Creative Commons Attribution License, which permits unrestricted use, distribution, and reproduction in any medium, provided the original work is properly cited.

\begin{abstract}
Aim. The aim of this paper is to investigate the effects of ethyl pyruvate (EP) on experimental liver fibrosis induced by bile duct ligation (BDL) and explore the underlying molecular mechanisms. Material and Method. Rats were randomly divided into three groups: the sham group, the BDL group, and the BDL+EP group. Liver fibrosis was induced by common bile duct ligation and was evaluated by serum biochemical parameter levels, Masson's trichrome staining, $\alpha$-SMA expression, and collagen I deposition. The levels of Nrf2 signaling pathway-related antioxidant genes (Nrf2, SOD2, NQO1, and GSH-Px) in liver tissues were also measured. Meanwhile, the mRNA expression levels of HMGB1, IL-1 $\beta$, TNF- $\alpha$, and HSP27 were analyzed. In BDL-induced liver fibrosis rats, the successfully established model was confirmed by the significant increase of serum ALT and AST levels, the high liver fibrosis score, $\alpha$-SMA expression, and collagen deposition. Results. Compared with the BDL group, EP administration could diminish fibrosis level and substantially increase the expression of Nrf2 signaling pathway-related antioxidant genes. Furthermore, EP significantly suppressed the mRNA expression levels of HMGB1, IL-1 $\beta$, TNF- $\alpha$, and HSP27. Conclusions. The results suggested that EP administration could effectively inhibit the liver fibrosis induced by BDL in rat, which may be associated with the enhanced activity of Nrf2 to mediate antioxidant enzyme system and downregulate the inflammatory genes.
\end{abstract}

\section{Introduction}

Liver fibrosis is a common result of repeated hepatic injury, which is characterized by the deposition of extracellular matrix (ECM). Activated hepatic stellate cells (HSCs) are the major sources of myofibroblasts and the main ECMproducing cells in the fibrogenic process [1-4]. Reactive oxygen species (ROS) has been confirmed as a key step in onset and progression of liver fibrosis [5], which not only causes excessive damage to hepatocytes but also significantly leads to HSC activation [6, 7]. Previous studies suggested that ROS contributed to HSC activation via mediating cytokine. Recently, it was reported that superoxide anion $\left(\mathrm{O}_{2}{ }^{-}\right)$, acetaldehyde, and arachidonic acid could stimulate HSCs. Moreover, ROS could promote early growth response factor-1 expression on protein and mRNA levels, which was a promoter of inflammation in cholestatic liver fibrosis.

Nuclear factor erythroid-2-related factor 2 (Nrf2) is a key transcription factor that regulates antioxidant enzymes and phase 2 detoxifying. Upon exposure to oxidative or 
electrophilic stress, cytosolic Nrf2 is phosphorylated and translocated to the nucleus, where it binds to the antioxidant response elements (AREs) by interacting with transcription factors in the leucine zipper region (bZIP) family $[8,9]$ and leads to an array of Nrf2 signaling pathway-related genes, such as heme oxygenase-1 (HMOX1), NADPH quinone oxidoreductase 1 (NQO1), catalase (CAT), superoxide dismutase (SOD), and glutathione peroxidase (GSH-Px) [10-12]. These antioxidant enzymes play important roles in the elimination of ROS.

Ethyl pyruvate (EP), a lipophilic derivative of pyruvate, is safer and more stable than pyruvate, but affords protective effects of pyruvate. EP has been widely accepted as a protective agent and shown to be safe in clinical doses. Its protective effects are attributed to its antioxidant properties $[13,14]$, anti-inflammatory $[13,15]$, and antiapoptotic effects $[16,17]$. EP can effectively scavenge $\mathrm{H}_{2} \mathrm{O}_{2}, \mathrm{O}_{2}{ }^{-}$, and $\mathrm{OH}^{-}$. Moreover, EP can inhibit the release of high-mobility group box 1 (HMGB1) [17], interleukin-1 $\beta$ (IL-1 $\beta$ ), and tumor necrosis factor-alpha (TNF- $\alpha$ ). Thus, we suppose that whether EP exerts protective effects and prevents the progression of liver fibrosis.

\section{Materials and Methods}

2.1. Animals and Experimental Design Methods. Male Sprague-Dawley rats $(250 \mathrm{~g}-280 \mathrm{~g})$ were obtained from DaShuo (Chengdu, China), and the Institutional Animal Care and Use Committee of Chengdu Medical College approved the experimental protocol. All rats were housed in plastic cages ( 4 rats per cage) with maintained conditions $\left(20-22^{\circ} \mathrm{C}, 54 \%\right.$ humidity, and $12 \mathrm{~h}$ light/dark cycle) and had free access to a standard rodent diet and water. The rats were acclimatized for 1 week prior to use.

EP was purchased from Solarbio (Beijing, China; purity 98\%). Animals were randomly assigned to three groups: the sham group $(n=15)$, the BDL group $(n=18)$, and the BDL+EP group $(n=18)$. In the sham group, rats underwent a laparotomy without common bile duct ligation (BDL). In the BDL group, rats underwent $\mathrm{BDL}$ to develop liver fibrosis. In the $\mathrm{BDL}+\mathrm{EP}$ group, rats underwent BDL and were intraperitoneally injected with EP diluted by Ringer's lactate solution $(40 \mu \mathrm{g} / \mathrm{ml} ; 40 \mathrm{mg} / 1000 \mathrm{~g}$ per day). Based on the process of liver fibrosis induced by BDL, each group was further divided into 3 subgroups (2, 4, and 6 weeks, respectively; for the sham subgroups: $n=5$; for the BDL subgroups: $n=6$; for the BDL+EP subgroups: $n=6$ ). BDL operation was achieved under general anesthesia. Laparotomy was made, and the common bile duct was localized, doubly ligated, and cut between these two ligatures [18].

2.2. Model. When rats under deep anesthesia were sacrificed, laparotomy was made, while the liquid in abdominal cavity was collected for volume measurement. The blood was collected from the heart, followed by removing and weighting liver tissues immediately. The sections from the left liver lobe were cut into several pieces. Some of them were fixed in $10 \%$ buffered neutral formalin; others were frozen at $-80^{\circ} \mathrm{C}$ for mRNA and protein detection.

According to the following formula [19], the liver index was calculated: Liver index $=($ liver weight $/$ rat weight $) \times 100 \%$.

2.3. Serum Biochemistry Analysis. Blood samples were obtained and separated by centrifugation (3000× g, $15 \mathrm{~min}$ ) to collect serum. Alanine aminotransferase (ALT) and aspartate aminotransferase (AST) levels were measured using an Auto Chemistry Analyzer (Hitachi, Japan). The test reagents were obtained from Jiancheng (Nanjing, China).

2.4. Tissue Histopathological Examination of Liver Sections. Fixed tissues were embedded in paraffin, sectioned, deparaffinized, and rehydrated. Sections were stained with hematoxylin and eosin (H\&E) for histological examination and Masson's trichrome for collagen deposition. According to the score standard proposed by Thompson [19], fibrosis was scored semiquantitatively as follows. Score 0: absent; Score 1: trace, slender septa present; Score 2: mild, slender septa linking hepatic veins; Score 3: moderate, broad or well-developed septa; Score 4: severe cirrhosis. Six fields were taken per liver section to obtain the mean value.

2.5. Immunohistochemistry. For immunohistochemistry, fixed liver sections ( $5 \mu \mathrm{m}$ thick) were deparaffinized, rehydrated, blocked with $0.3 \%$ nonspecific catalase, antigen retrieved with high pressure, and blocked with $10 \%$ nonspecific goat serum enzyme. Subsequently, the sections were incubated in polyclonal anti-rat antibody $\alpha$-SMA ( $\alpha$-smooth muscle actin, diluted 1:200; Proteintech), Nrf2 (diluted $1: 100$; Proteintech), SOD2 (diluted 1:100; Proteintech), Hsp27 (diluted 1:100; Proteintech), and HMGB1 (diluted 1:200; Proteintech). After being washed in phosphatebuffered saline (PBS), the sections were incubated with the Polink-1-HRP-DAB Detection System to rabbit antibody for $1 \mathrm{~h}$ and visualized using DAB. Finally, the positive expressions of $\alpha$-SMA, Nrf2, SOD2, Hsp27, and HMGB1 were observed at 400x under a light microscope (Olympus BX 53, Japan). Ten positive areas were randomly taken and analyzed using Image-Pro Plus 6.0 software.

2.6. Immunofluorescence. The frozen liver tissues were cut into sections ( $15 \mu \mathrm{m}$ thick) and fixed with methanol. Sections were pretreated previously, incubated in the mixture of antiCK19 and anti-Nrf2 antibodies (anti-CK19 antibody, rabbit, $1: 150$, BioTECH; anti-Nrf2 antibody, mouse, $1: 100$, Santa Cruz, USA) overnight at $4^{\circ} \mathrm{C}$. Then, sections were washed with PBS, incubated with fluorescence secondary antibody (anti-mouse Alexa Fluor 488-conjugated, 1:100, green; anti-rabbit Alexa Fluor 594-conjugated, $1: 100$, red; Proteintech) for $2 \mathrm{~h}$ at $37^{\circ} \mathrm{C}$. Next, sections were rinsed with PBS and mounted by DAPI. All sections were photographed, and the positions of CK19 and Nrf2 were observed with a light microscope (Olympus/BX51, Tokyo, Japan) at 400x. The images were analyzed using Image-Pro Plus 6.0 software.

2.7. Western Blot Analysis. According to the standard protocol, $80 \mu \mathrm{g}$ total protein samples were prepared and determined by BCA assay kit (KeyGEN). Then, $20 \mu \mathrm{g}$ total 
TABLE 1: Primers for q-RTPCR.

\begin{tabular}{lrr}
\hline Gene & Forward primer sequence & Reverse primer sequence \\
\hline$\alpha$-SMA & TTATTGCTCCTCCAGAAC & CTTCGTCATACTCCTGTT \\
HSP27 & AGGATGGCGTGGTGGAGA & GGGAGGAGGAAACTTGGGTG \\
Nrf2 & CGCCGCCTCACCTCTGCTGCCAGTAG & AGCTCATAATCCTTCTGTCG \\
Actin & TATGGAATCCTGTGGCATC & GTGTTGGCATAGAGGTCTT \\
TNF- $\alpha$ & TGGCGTGGAGCTGAGAGA \\
Colla1 & TCAGAACATCACCTACCA & GCAATGATCCCAAAGTAGACCT \\
IL-1 & GCAGAATGACAGCCTTAT & GCAGAATGACAGCCTTAT \\
HMGB1 & GGCGGCTGTTTTGTTGACAT & TCAGAACATCACCTACCA \\
\hline
\end{tabular}

proteins were separated by SDS-polyacrylamide gel, transferred to a PVDF membrane (KeyGEN), and blocked with $5 \%$ BSA in Tris-buffered saline containing 0.5\% Tween 20, followed by incubation with primary (polyclonal rabbitanti-rat SOD2 antibody, ZSGBBIO, China, 1:1000) and secondary antibody (goat-anti-rabbit antibody, ZSGBBIO, China, $1: 8000$ ). $\beta$-Actin (primary antibody, $1: 200$; secondary antibody, 1:6000; Santa Cruz Biotechnology, Inc., Santa Cruz, CA, USA) was used as an internal control. Protein bands were visualized using chemiluminescence reagent (KeyGEN).

2.8. ELISA Assay for Nrf2. Liver tissues were homogenized and centrifuged at $4^{\circ} \mathrm{C}(5000 \times \mathrm{g} / 10 \mathrm{~min})$ in PBS buffer with protease inhibitors. Then, protein concentration was determined by a BCA method. The ELISA kit for Nrf2 was performed according to the protocol provided by the manufacturer (Hongju, Shanghai, China). Finally, when the reaction stopped, the optical density of each well was determined at $450 \mathrm{~nm}$.

2.9. Real-Time Reverse Transcriptase-Polymerase Chain Reaction Analyses. Total RNA in liver tissues was isolated with TRIzol reagent (DBI Bioscience, Germany). Then, $2 \mu \mathrm{g}$ of total RNA was reverse-transcribed into cDNA using a MultiScribe $^{\mathrm{TM}}$ Reverse Transcriptase (DBI Bioscience). With the PCR Thermal Cycler (Bio-Rad, USA), gene expression was measured in a standard protocol with SYBR Green PCR Master Mix x (DBI Bioscience). Quantitative RT-PCR was performed at least three times, and the primer sequences are summarized in Table 1. Samples were analyzed in triplicate according to the Delta-Delta threshold ( $\Delta \Delta \mathrm{Ct})$ method, which was used to quantify gene expression levels.

2.10. Statistical Analysis. SPSS 17.0 software was used for statistical analysis. Quantitative data were expressed as mean \pm standard error of the mean (SEM) and subjected to one-way analysis of variance (ANOVA). $P<0.05$ was considered as significant.

\section{Results}

3.1. EP Ameliorated BDL-Induced Hepatic Injury. The structure of the liver tissues was completely maintained and remained ordered in the sham group. Disordered lobular structure and bile duct epithelial hyperplasia were observed in the $\mathrm{BDL}$ and $\mathrm{BDL}+\mathrm{EP}$ groups. However, the administration of EP reduced liver pathophysiology features in the BDL+EP group compared with the BDL group (Figure 1(a)).

Morphological analysis of liver sections stained with Masson's was performed. We observed a normal morphology, with scarce ECM deposition in the sham group. The BDL group showed an altered morphology, with thick collagen bundles. In contrast, the $\mathrm{BDL}+\mathrm{EP}$ group showed lower ECM deposition (Figure 1(b)). Liver fibrosis score indicated the progression of liver fibrosis. The liver fibrosis score was lower in the BDP+EP group at 2 and 4 weeks compared with the BDL group (Figure $1(\mathrm{c}), P<0.01$ ). These observations indicated that EP could postpone the liver fibrosis progression.

The biochemical analysis results are presented in Figure 1(c). Serum AST and ALT levels were significantly higher in the BDL group compared to the sham group $(P<0.01)$. EP administration significantly reduced ALT and AST levels in liver fibrosis rats compared to the BDL group $(P<0.05)$. These observations suggested that EP could effectively prevent rat liver from damage.

3.2. Effects of EP on $\alpha$-SMA Expression and ECM. With immunohistochemistry staining, the $\alpha$-SMA and collagen I protein expression localized predominantly in the fibrous septa, inflamed area, and portal area. In normal liver tissue, ordered collagen I expression existed; its expression was evident in the sham group. In contrast, more disordered collage I expression appeared in the $\mathrm{BDL}$ and $\mathrm{BDL}+\mathrm{EP}$ groups (Figures 2(a) and 2(b)). A higher positive number of $\alpha$-SMA was detected in the BDL group than that in the sham group, while the expression level of $\alpha$-SMA in the BDL+EP group was significantly lower than that in the BDL group, especially at 2 and 4 weeks (Figure $2(c), P<0.05$ ). The results of RT-PCR showed that mRNA level of collagen I was higher in the BDL and BDL+EP groups compared with the sham group. However, after EP administration, mRNA level of collagen I significantly reduced compared with the BDL group (Figure $2(\mathrm{~d})$ ).

3.3. Effects of EP on Inflammatory Gene Expression. The immunohistochemistry-positive expression level of HMGB1 was analyzed in the present experiment. Compared with the sham group, HMGB1 showed a higher expression in liver tissue in the BDL group at 2, 4, and 6 weeks (Figure 3(a), $P<0.05)$. 


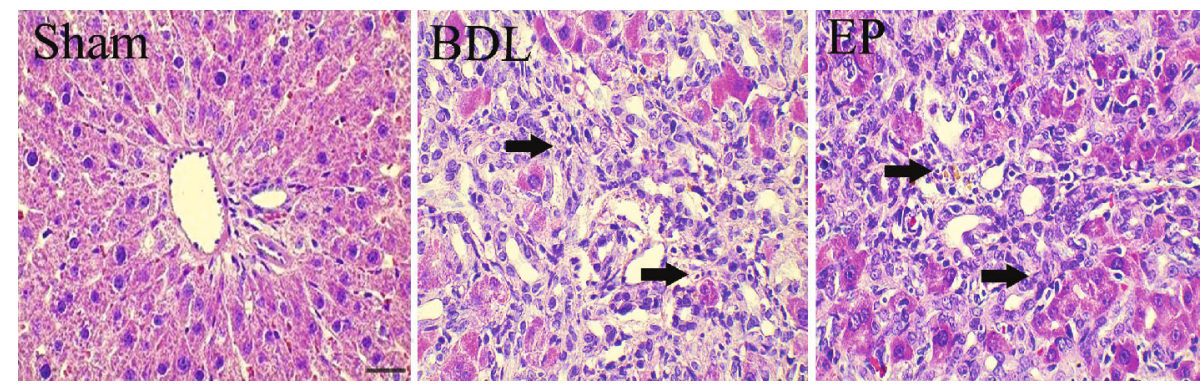

(a)

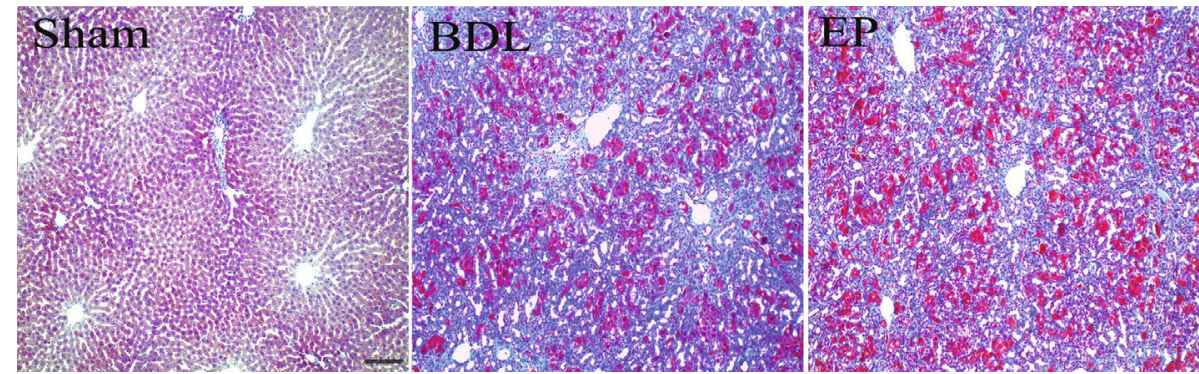

(b)
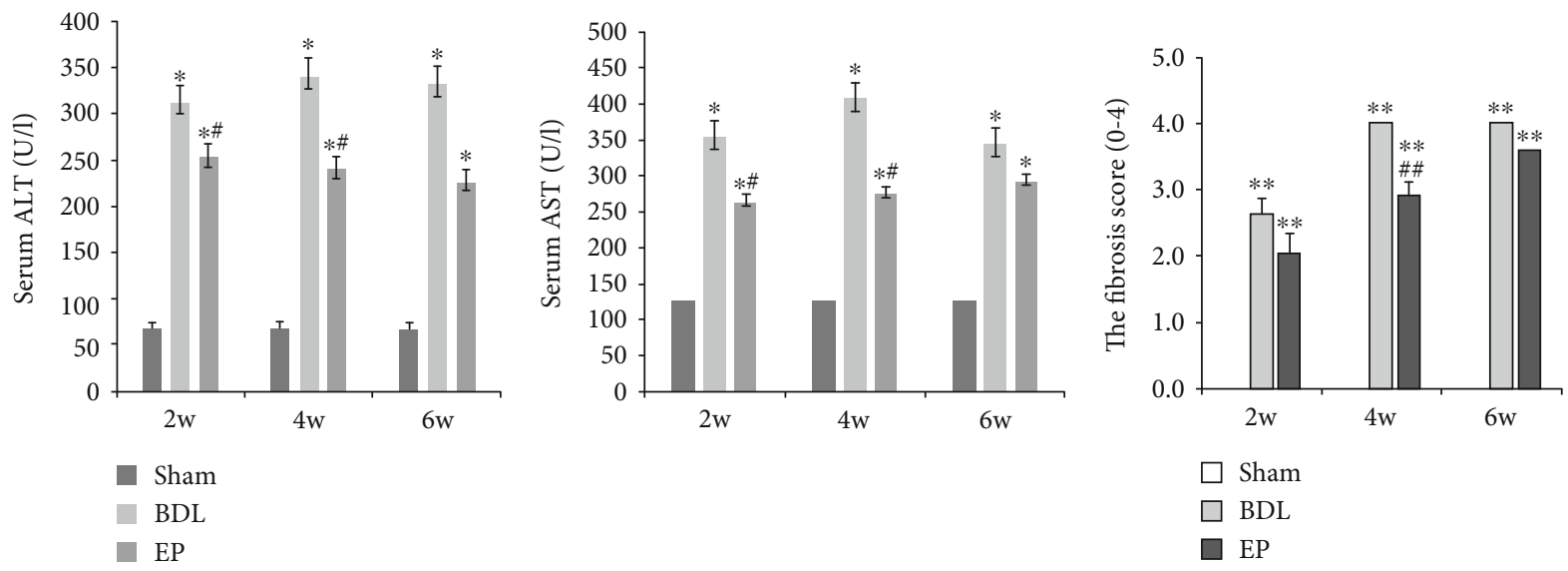

(c)

FIgURE 1: EP improved liver function in rats. (a) HE staining $(\times 400)$. (b) Masson's trichrome staining at 4 weeks $(\times 100)$. (c) The serum ALT and AST levels and the semiquantitation of hepatic fibrosis using pathological scoring with Masson's trichrome staining ( $*$ : vs. sham group, ${ }^{*} P<0.05,{ }^{* *} P<0.05$; \#: vs. BDL group, ${ }^{\#} P<0.05,{ }^{\# \#} P<0.01, n=6$ per group). Scale bars $=20 \mu \mathrm{m}$; “ $\rightarrow$ " indicates the positions of disordered lobular structure and bile duct epithelial hyperplasia.

The RT-PCR relative expressions of HMGB1, IL-1 $\beta$, and TNF- $\alpha$ were analyzed. EP treatment significantly diminished HMGB1 expression, and lower levels were observed in the BDL+EP group compared to the BDL group, especially at 4 and 6 weeks (Figure $3(\mathrm{~b}), P<0.05$ ). Compared to the sham group, the IL- $1 \beta$ and TNF- $\alpha$ expression levels significantly increased in the BDL group. However, EP treatment inhibited their increase, while lower levels were shown in the BDL+EP group than those in the BDL group at 2, 4, and 6 weeks (Figures 3(c) and 3(d), $P<0.05)$. The results indicated that EP could downregulate the inflammatory response.

3.4. Effects of EP on Nrf2 and HSP27 Expression. With HSP27 and Nrf2 immunohistochemistry, the cytolymph-positive cells were observed in the sham group, while more cytolymph and nucleus-positive cells appeared in the BDL and BDL+EP groups (Figures 4(a) and 4(b)). The immunofluorescence double staining showed that Nrf2 protein expressed in hepatocyte and biliary epithelium cells (Figure 4(c)).

With mRNA analyses, we noticed that HSP27 presented a higher level in the BDL group than that in the sham group at 2, 4, and 6 weeks (Figure 4(d), $P<0.05$ ). However, EP reversed the enhancement of HSP27 compared to the BDL group, especially at 4 weeks (Figure $4(\mathrm{~d}), P<0.05$ ). We noticed that Nrf2 expression level was higher in the BDL group than that in the sham group at 2, 4, and 6 weeks. Compared to the BDL group, EP administration increased the Nrf2 levels in liver fibrosis rats at 2 and 4 weeks (Figure 4(d), $P<0.05$ ). 


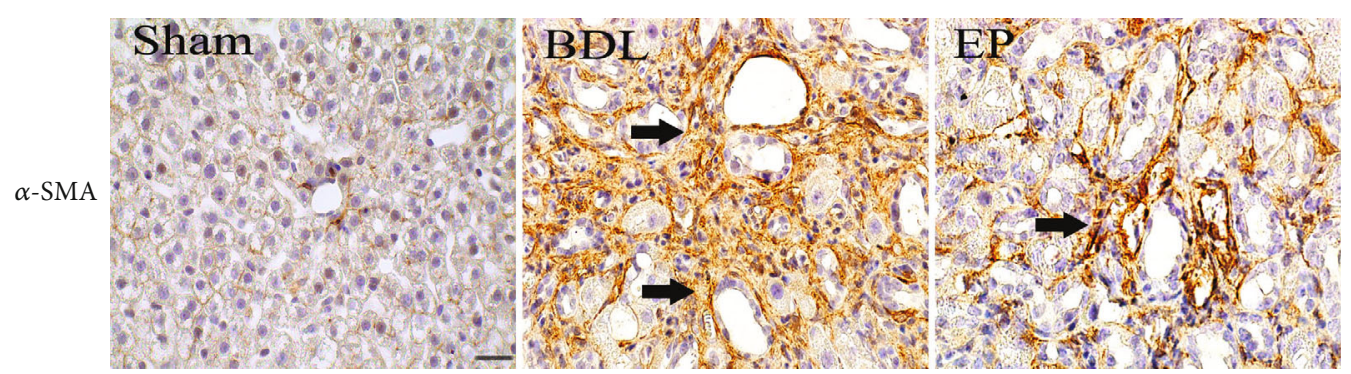

(a)

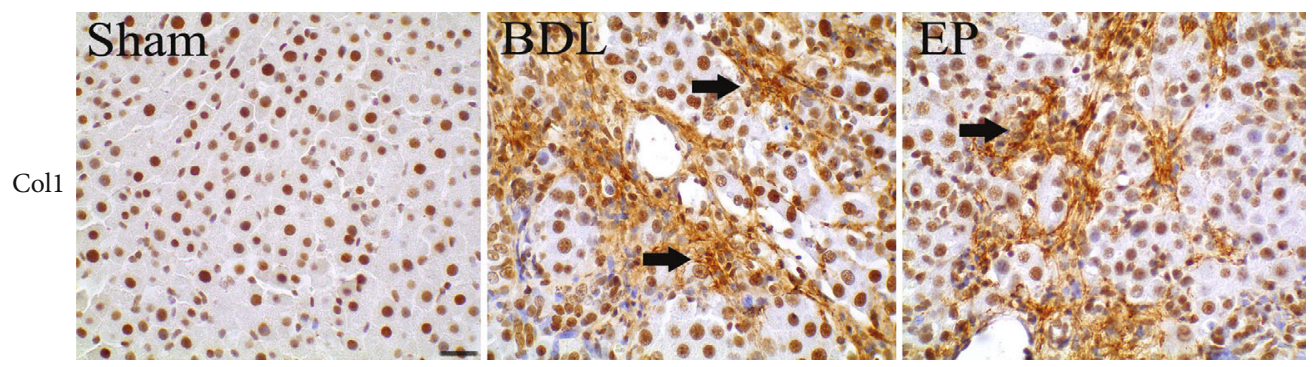

(b)
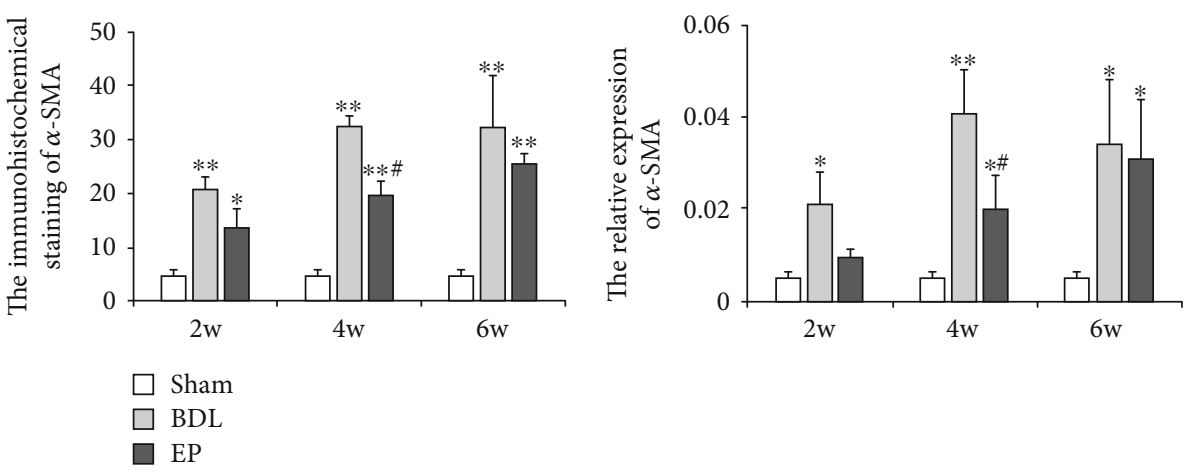

(c)

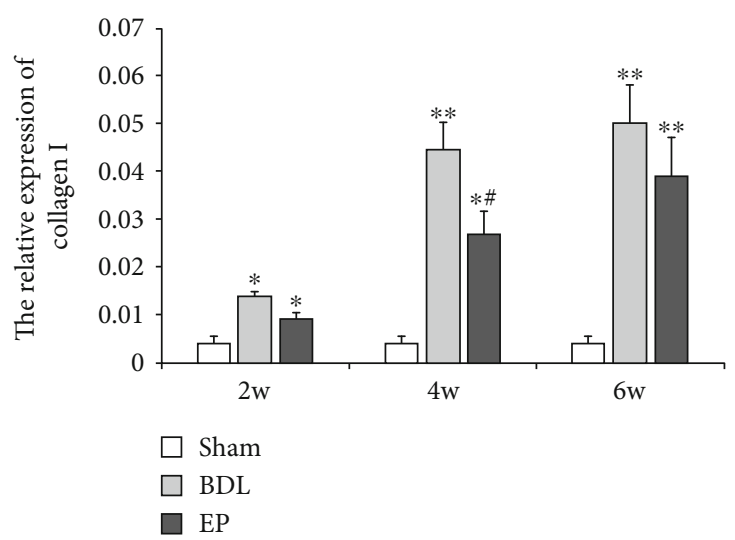

(d)

FIgURE 2: Detection of $\alpha$-SMA and collagen I in the liver. (a) Hepatic stellate cell staining of $\alpha$-SMA. The immunohistochemistry of $\alpha$-SMA at 4 weeks $(\times 400)$. (b) Hepatic stellate cell staining of collagen I. (c) Analysis of $\alpha$-SMA-positive staining in liver sections. (d) Analysis of collagen I mRNA expression in liver tissue (*: vs. sham group, ${ }^{*} P<0.05,{ }^{* *} P<0.05$; \#: vs. BDL group, ${ }^{\#} P<0.05,{ }^{\# \#} P<0.01, n=6$ per group). Scale bars $=20 \mu \mathrm{m} ; "$ " $\rightarrow$ " indicates the positions of $\alpha$-SMA or collagen I.

The quantitative analysis of Nrf2 protein was performed with ELISA detection. Nrf2 protein in the BDL group showed a higher expression compared to the sham group $(2,4$, and 6 weeks, $P<0.05$, respectively).
EP treatment markedly increased the Nrf2 protein expression; it had a higher level in the BDL+EP group than that in the BDL group, especially at 4 weeks (Figure 4(d), $P<0.05)$. 


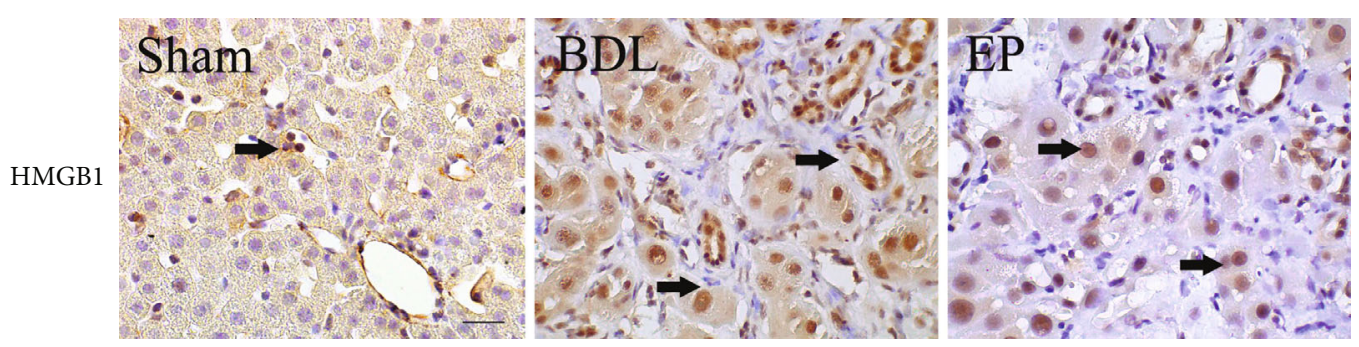

(a)

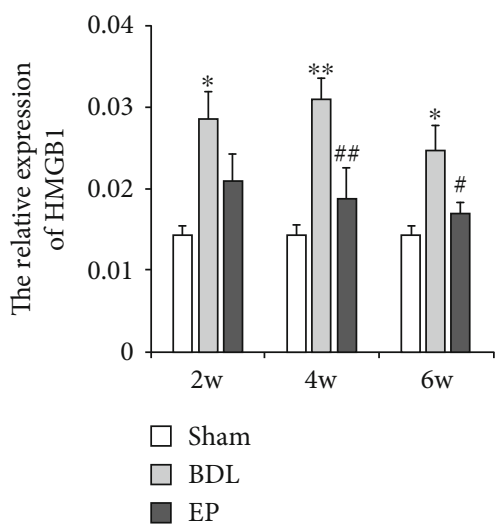

(b)

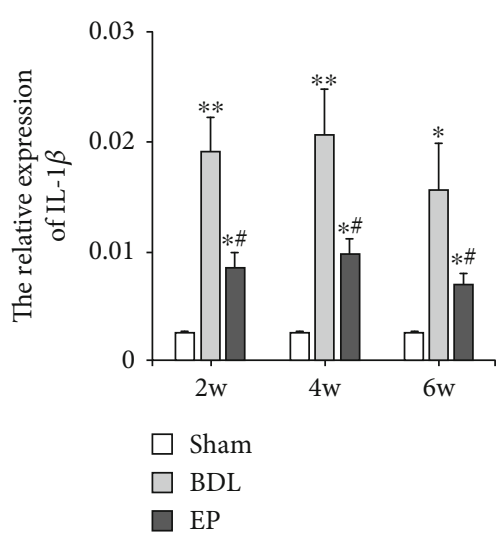

(c)

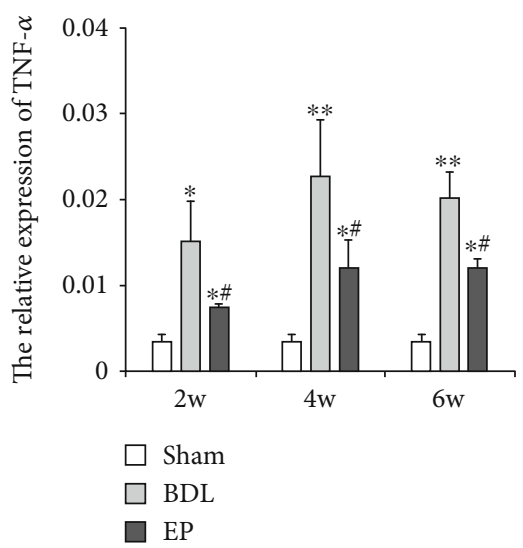

(d)

Figure 3: The HMGB1, IL-1 $\beta$, and TNF- $\alpha$ expression. (a) Immunohistochemical staining of HMGB1 in the rat livers $(\times 400)$ at 4 weeks. (b, c, d) HMGB1, IL-1 $\beta$, and TNF- $\alpha$ mRNA expression levels in liver tissue, respectively. (*: vs. sham group, ${ }^{*} P<0.05$, ${ }^{* *} P<0.05$; \#: vs. BDL group, ${ }^{\#} P<0.05,{ }^{\# \#} P<0.01, n=6$ per group). Scale bars $=20 \mu \mathrm{m}$; “ $\rightarrow$ " indicates the positions of HMGB1.

3.5. Effects of EP on Nrf2 Signaling Pathway-Related Gene Expression. With the immunohistochemistry SOD2, the cytolymph-positive cells were observed in the sham group, while more cytolymph and nucleus-positive cells appeared in the BDL and BDL+EP groups (Figure 5(a)). The mRNA expression levels of Nrf2 signaling pathway-related genes (SOD2, NQO1, and GSH-Px) were analyzed. We noticed that SOD2 expression level was higher in the BDL group than that in the sham group at 2, 4 , and 6 weeks. Compared to the BDL group, EP administration increased the SOD2 level in liver fibrosis rats at 2 and 4 weeks (Figure 5(b), $P<0.05$ ). In contrast, the NQO1 and GSH showed lower levels in the BDL group than those in the sham group, and higher levels was observed in the BDL+EP group than those in the BDL group, especially at 4 weeks (Figures 5(d) and 5(e), $P<0.05)$. The SOD2 protein was detected by the western blot, which presented a similar trend with its mRNA expression (Figure 5(c), $P<0.05$ ). These data suggested that EP could enhance the SOD2 protein expression. All the above results indicated that EP could increase the expression of Nrf2 signaling pathway-related genes.

3.6. Experiment Mechanism Diagram. In hepatoprotective applications, EP mainly focused on preventing and attenuating oxidative and inflammatory damage to the liver. In the present paper, EP played a protective role against liver fibrosis mainly on antioxidation. The diagram of mechanism showed the postulation of HSP27 regulating the Nrf2 activation (Figure 6). HSP27 might affect the banding of Nrf2 and AREs via interacting with JUN, which led to the decrease of
Nrf2 activation. The increased oxidative stress condition led to the extracellular release of reduced form of acetylated HMGB1 by necrotic cell death, which acted as inflammatory cytokine. The active secretion of HMGB1 by the activated macrophages and monocyte cells was reported to occur in response to the inflammatory stimuli of various cytokines, i.e., TNF- $\alpha$, IL-1, and IFN- $\gamma$.

\section{Discussion}

The present study demonstrated that EP could inhibit the liver fibrosis progression in rats, which was indicated by the improvements of serum ALT and AST levels and liver fibrosis contents. The effects of EP inhibiting the liver fibrosis process were associated with EP enhancing the Nrf2 signaling pathway-related antioxidant protein expression and decreasing the inflammatory factor expressions.

EP had received interest as its hepatoprotective effects in fatty liver, hepatic ischemia-reperfusion injury, and acuteon-chronic liver failure [20]. EP downregulated the expression of multiple proinflammatory proteins, including IL- $1 \beta$, TNF- $\alpha$, and HMGB1 in animal experiments of endotoxemia and sepsis [21]. As an endogenous danger signal molecule, HMGB1 could induce various proinflammatory cytokines to secrete and aggravate inflammatory processes [22].

Previous researches reported that HMGB1 was closely involved in fibrotic disorders including cystic fibrosis, liver fibrosis, and pulmonary fibrosis [23, 24]. Transforming growth factor-beta (TGF- $\beta$ ) family of cytokines could also 


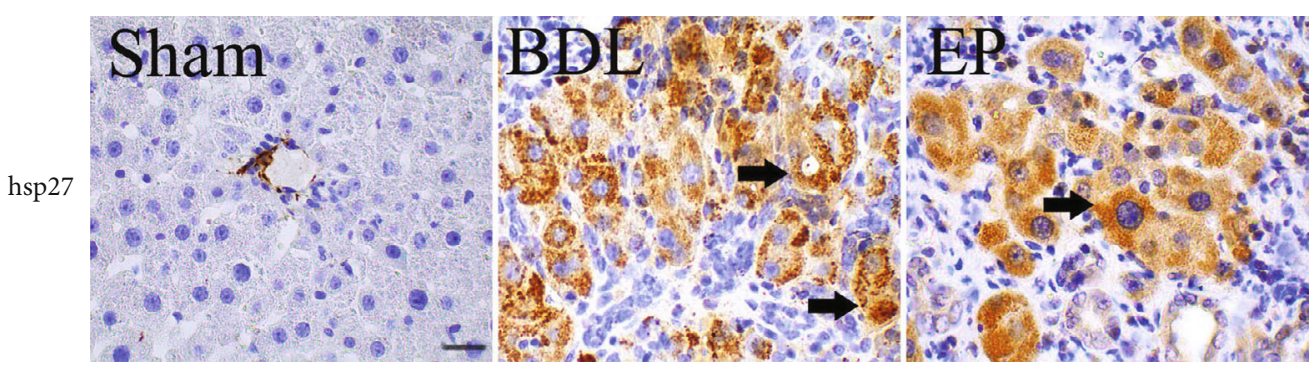

(a)

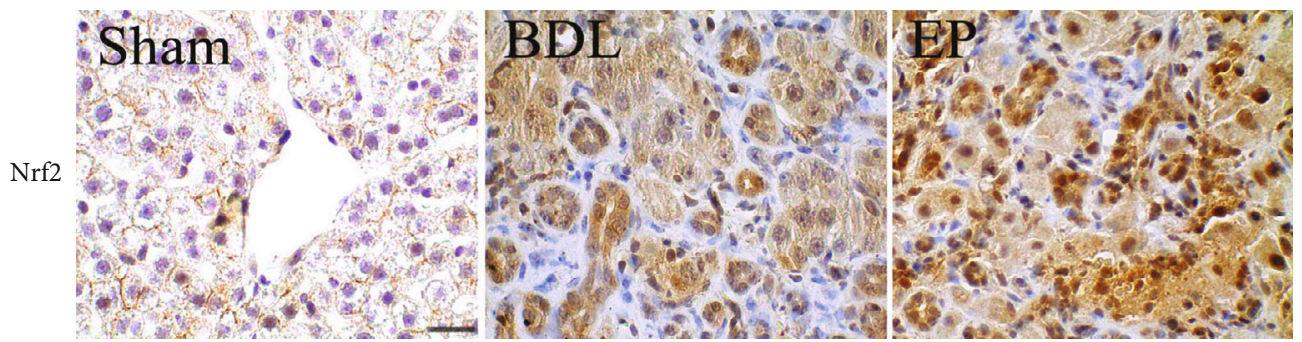

(b)
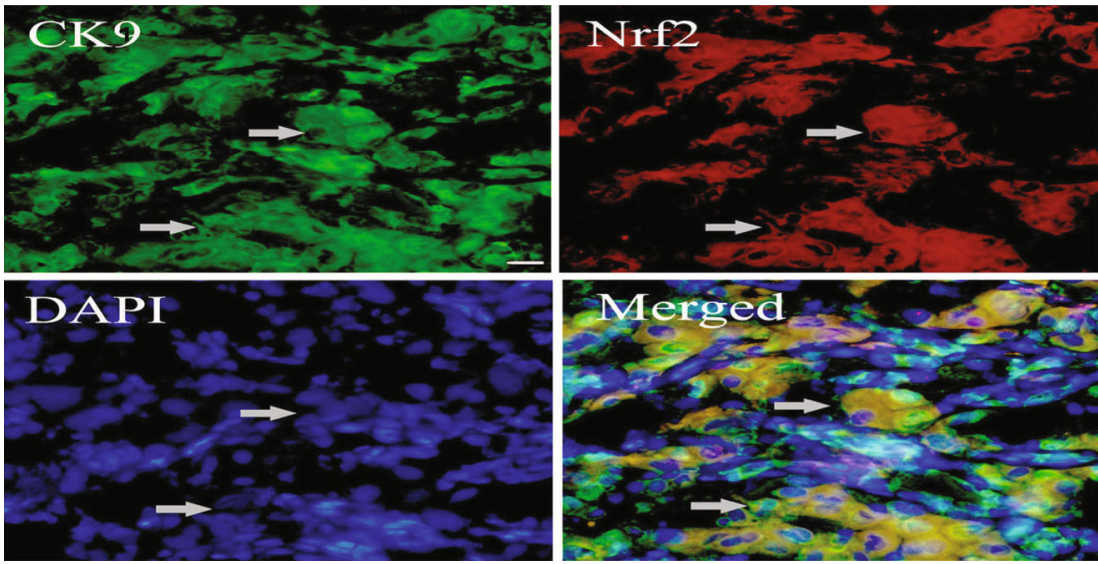

(c)
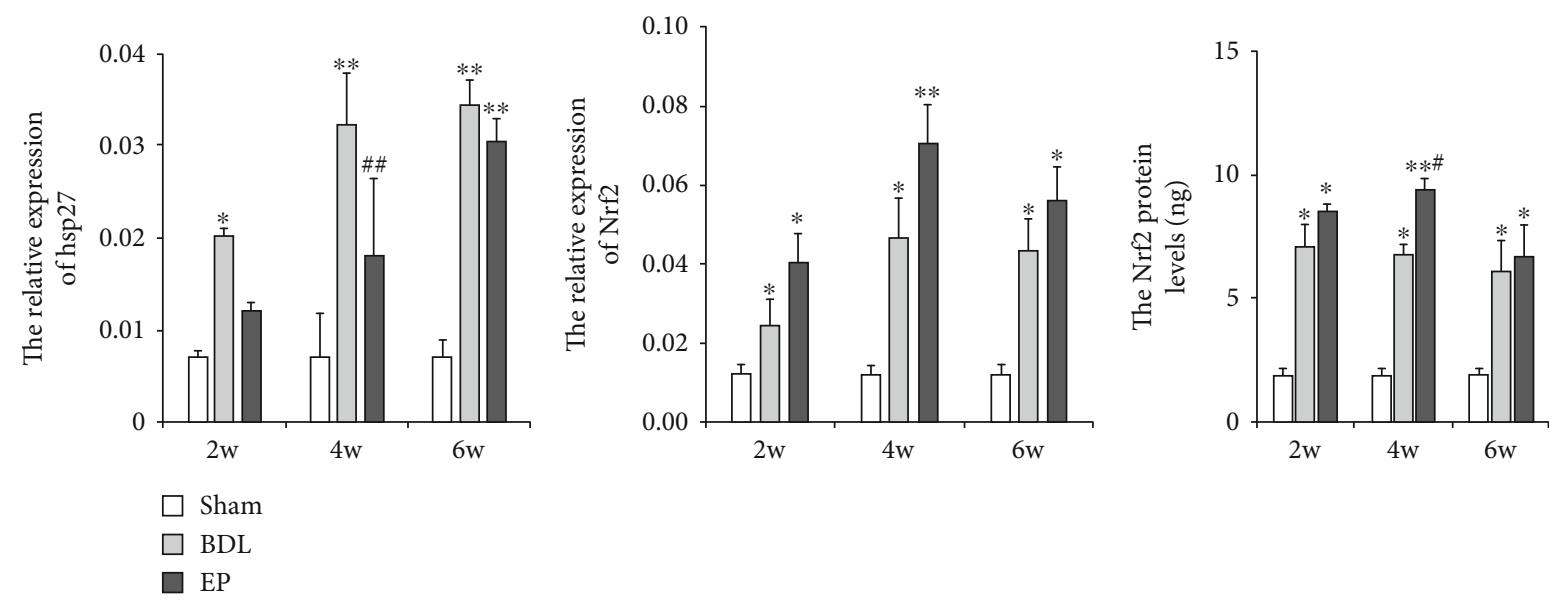

(d)

Figure 4: Effects of EP on Nrf2 and HSP27. (a) Immunohistochemical staining of Nrf2 in the rat livers $(\times 400)$. Scale bars $=20 \mu$ m; “ $\rightarrow$ " indicates the positions of Nrf2. (b) Immunohistochemical staining of HSP27 in the rat livers $(\times 400)$. Scale bars $=20 \mu \mathrm{m}$. $(\mathrm{c})$ The immunofluorescence double staining for Nrf2/CK19. CK19 was stained with green color, Nrf2 was stained with red color, nucleus was stained by DAPI with blue color, and the coexpression of CK19 with Nrf2 within the same cell showed yellow color (merged). Scale bars $=20 \mu \mathrm{m}$; “ $\rightarrow$ " indicates the positions of CK19/Nrf2/DAPI/merged. (d) Analysis of HSP27 and Nrf2 mRNA expression. The Nrf2 protein level detected by ELISA. (*: vs. sham group, ${ }^{*} P<0.05,{ }^{* *} P<0.05$; \#: vs. BDL group, ${ }^{\#} P<0.05,{ }^{\#} P<0.01, n=6$ per group). 


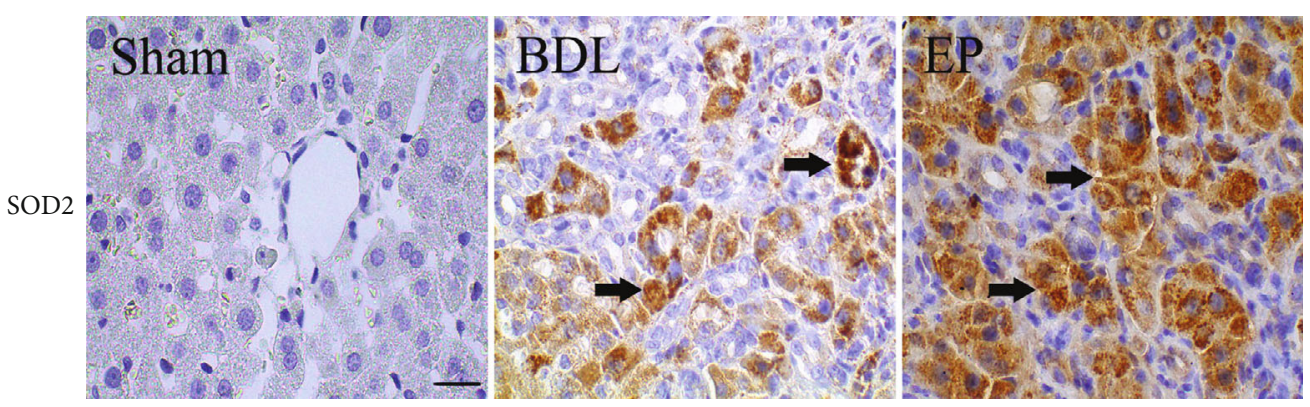

(a)

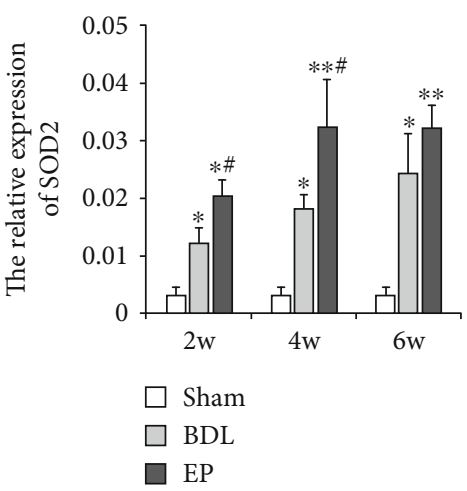

(b)

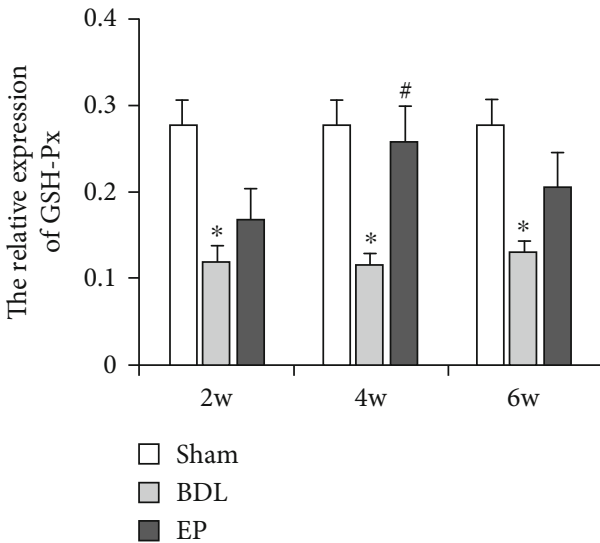

(d)

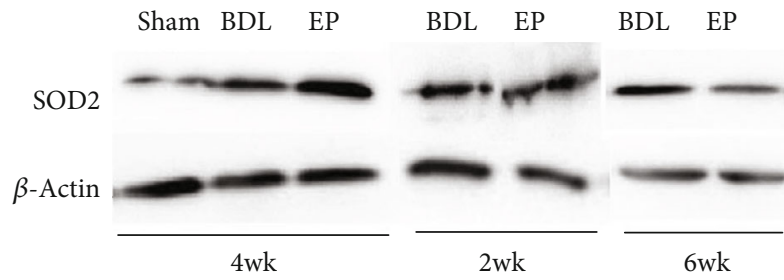

(c)

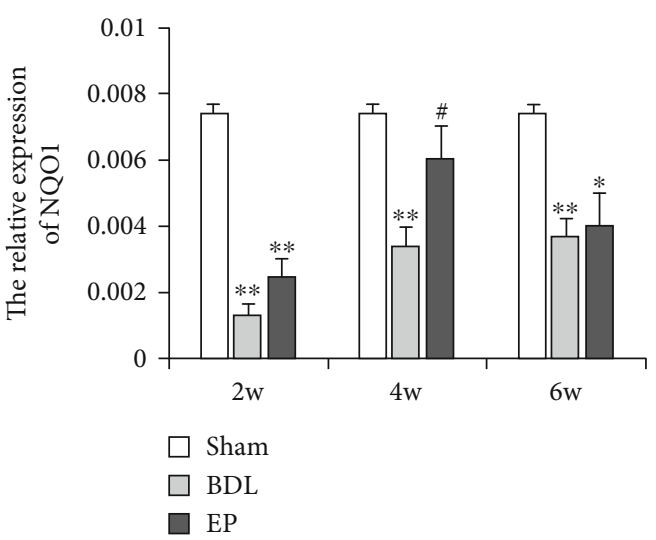

(e)

FIgURE 5: Nrf2 signaling pathway-related genes expression. (a) Immunohistochemical staining of SOD2 in the rat livers ( $\times 400)$. (b) The analysis of SOD2 mRNA expression. (c) Western blots for SOD2. (d, e) NQO1 and GSH-Px mRNA expression levels in liver tissue, respectively. (*: vs. sham group, ${ }^{*} P<0.05,{ }^{* *} P<0.05$; \#: vs. BDL group, ${ }^{\#} P<0.05,{ }^{\# \#} P<0.01, n=6$ per group). Scale bars $=20 \mu \mathrm{m}$, " $\rightarrow$ " indicates the positions of SOD2.

been driven by HMGB1 in renal fibrosis [25], while proinflammatory cytokines and angiogenic factors could directly stimulate the HSC activation in liver fibrosis. Our results showed that EP could inhibit HSC activation as EP significantly downregulated the HMGB1 level at 4 and 6 weeks of liver fibrosis induced by BDL, while IL- $1 \beta$ and TNF- $\alpha$ levels decreased substantially.

Moreover, a number of reports showed that the antiinflammatory effect of EP was attributable to the inhibition of ROS-dependent signal transducer and activator of transcription (STAT) signaling [26, 27]. The antioxidative and anti-inflammatory effects of EP were also presented in the current study. It was generally accepted that oxidative stress and inflammation played important roles in the onset and development of liver fibrosis. Nrf2 is a central regulator of antioxidative response element-mediated gene expression. Ample evidences demonstrated that the high expression of Nrf2 was observed in detoxification organs, especially in the liver. By interacting with the ARE, Nrf2 induced a variety of downstream target expression against oxidative stress to protect hepatic cell from oxidative damage during development of common chronic liver diseases [28-30]. Caffeine and ginsenoside $\mathrm{Rg} 1$ had been shown to inhibit liver fibrosis through Nrf2-mediated induction of SOD, Nqo1, and GST [31-33]. Activation of Nrf2 might be a novel strategy to prevent or ameliorate toxin-induced liver injury and fibrosis. 


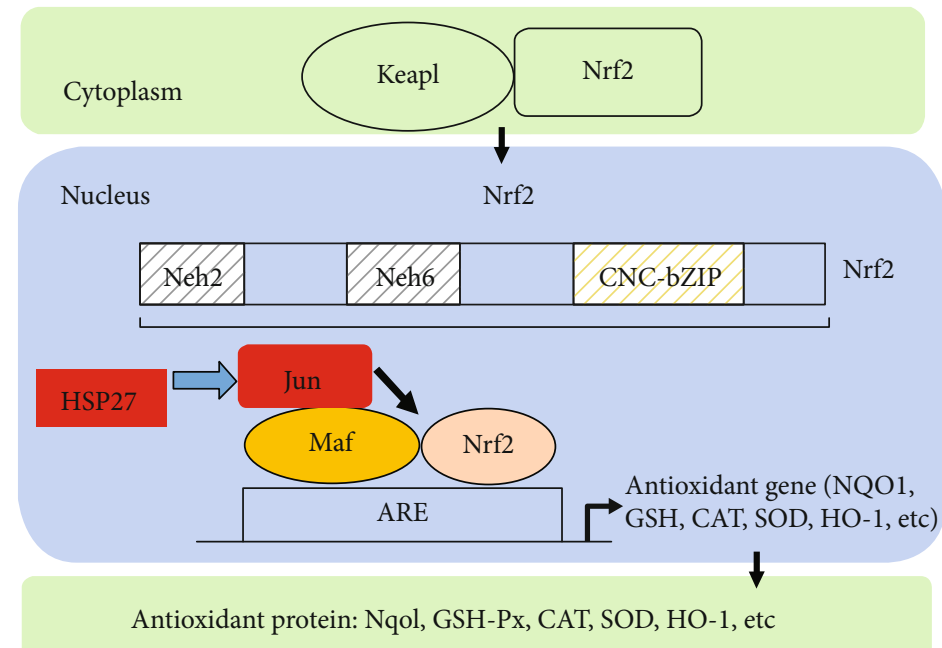

FIgURE 6: The diagram of postulation of HSP27 regulating the activating process of Nrf2. HSP27 might affect the banding of Nrf2 and AREs via interacting with JUN, which led to the decrease of Nrf2 activation.

EP was an effective ROS scavenger which could scavenge $\mathrm{H}_{2} \mathrm{O}_{2}$, another ROS and $\mathrm{OH}-$. Our results showed that EP could increase the protein expression levels of Nrf2 and SOD2, along with enhancing the levels of SOD2, Nqo1, and GSH-Px mRNA in the rat liver of BDL operation at 4 weeks. We observed that Nrf2 was spontaneously activated while comparing between the BDL group and the sham group. Moreover, Nrf2 activation was observed in hepatocytes and bile duct epithelial cells, and Nrf2 target genes SOD2, NQO1, and GSH-Px significantly increased after EP treatment. Interestingly, the increase of Nrf2 in the BDL+EP group had no significant differences compared with the BDL group at 2 weeks, while the elevation of SOD2 level had statistical difference. We considered that SOD2 was spontaneously activated and more sensitive when exposed to oxidative stress, and it might exponentially increase along with increasing Nrf2. The data indicated that EP had ability to enhance endogenous antioxidant capacity via promoting the Nrf2 pathway activation in liver fibrosis.

In the present study, EP also inhibited the enhancement of HSP27. Previous paper showed that there was a correlation between HSP27 and Nrf2 [34]. We found synchronous coexpression of HSP27, Nrf2, and JUN, while JUN participated in the Nrf2 activation process. We postulated that HSP27 might be involved in the regulation of Nrf2 activation. JUN could interact with bZIP of Nrf2, which had been considered as a key event for Nrf2 binding to AREs to initiate the Nrf2 signaling pathway-related genes. JUN includes c-JUN, JUN-D, and JUN-B, in which c-JUN can inhibit the combination of Nrf2 and AREs to reduce the Nrf2 activated [35]. Thus, HSP27 might affect the banding of Nrf2 and AREs via interacting with JUN, which led to Nrf2 activation and decreased the expression of Hsp27, a small HSP that could be induced by a wide variety of stresses including heat shock and oxidative stress [36].

In conclusion, EP exhibited significant protection against liver fibrosis as evidenced from the reversal of biochemical indices and histopathology. The protective effects of EP could be primarily due to its antioxidant and anti-inflammatory properties. However, the results suggested that EP showed significant protection in the advanced stage of liver fibrosis, but did not effectively prevent the liver from cirrhosis. More detailed studies are required.

\section{Data Availability}

No data were used to support this study.

\section{Conflicts of Interest}

The authors declare that they have no conflicts of interest.

\section{Authors' Contributions}

Yonghua Zong and Mingxiao Zhang participated in the design of this study, and they both performed the statistical analysis. Yonghua Zong carried out the study, and Mingxiao Zhang collected important background information and drafted the manuscript. All authors read and approved the final manuscript. Shuai Li and Wenqian Qi carried out the concepts, design, definition of intellectual content, literature search, data acquisition, data analysis, and manuscript preparation. Juan $\mathrm{Li}$ and Tonghua Liu provided assistance for data acquisition, data analysis, and statistical analysis. Chen Lu carried out literature search, data acquisition, and manuscript editing. Huijun Yang and Xiaosong Hu performed manuscript review. All authors have read and approved the content of the manuscript. Yonghua Zong and Mingxiao Zhang equally contributed to this work.

\section{Acknowledgments}

This work was supported by the Key Laboratory Project Fund of Sichuan Province (No. SYS10-002). 


\section{References}

[1] S. L. Friedman, "Mechanisms of disease: mechanisms of hepatic fibrosis and therapeutic implications," Nature Clinical Practice Gastroenterology \& Hepatology, vol. 1, no. 2, pp. 98105, 2004.

[2] D. A. Brenner, "Molecular pathogenesis of liver fibrosis," Transactions of the American Clinical and Climatological Association, vol. 120, pp. 361-368, 2009.

[3] R. Schwabe and R. Bataller, "Liver fibrosis. Foreword," Seminars in Liver Disease, vol. 35, no. 2, pp. 95-96, 2015.

[4] F. Duval, J. E. Moreno-Cuevas, M. T. Gonzalez-Garza, C. Rodriguez-Montalvo, and D. E. Cruz-Vega, "Liver fibrosis and protection mechanisms action of medicinal plants targeting apoptosis of hepatocytes and hepatic stellate cells," Advances in Pharmacological Sciences, vol. 2014, Article ID 373295, 11 pages, 2014.

[5] G. Poli, "Pathogenesis of liver fibrosis: role of oxidative stress," Molecular Aspects of Medicine, vol. 21, no. 3, pp. 49-98, 2000.

[6] A. I. Cederbaum, Y. Lu, and D. Wu, "Role of oxidative stress in alcohol-induced liver injury," Archives of Toxicology, vol. 83, no. 6, pp. 519-548, 2009.

[7] A. J. Czaja, "Hepatic inflammation and progressive liver fibrosis in chronic liver disease," World Journal of Gastroenterology, vol. 20, no. 10, pp. 2515-2532, 2014.

[8] C. Gorrini, I. S. Harris, and T. W. Mak, "Modulation of oxidative stress as an anticancer strategy," Nature Reviews Drug Discovery, vol. 12, no. 12, pp. 931-947, 2013.

[9] M. A. O'Connell and J. D. Hayes, "The Keap1/Nrf2 pathway in health and disease: from the bench to the clinic," Biochemical Society Transactions, vol. 43, no. 4, pp. 687-689, 2015.

[10] G. E. Mann, J. Niehueser-Saran, A. Watson et al., "Nrf2/ARE regulated antioxidant gene expression in endothelial and smooth muscle cells in oxidative stress: implications for atherosclerosis and preeclampsia," Acta Physiologica Sinica, vol. 59, no. 2, pp. 117-127, 2007.

[11] F. Bardag-Gorce, J. Oliva, A. Lin, J. Li, B. A. French, and S. W. French, "Proteasome inhibitor up regulates liver antioxidative enzymes in rat model of alcoholic liver disease," Experimental and Molecular Pathology, vol. 90, no. 1, pp. 123-130, 2011.

[12] E. O. Farombi, S. Shrotriya, H. K. Na, S. H. Kim, and Y. J. Surh, "Curcumin attenuates dimethylnitrosamine-induced liver injury in rats through Nrf2-mediated induction of heme oxygenase-1," Food and Chemical Toxicology, vol. 46, no. 4, pp. 1279-1287, 2008.

[13] M. P. Fink, "Ethyl pyruvate: a novel anti-inflammatory agent," Journal of Internal Medicine, vol. 261, no. 4, pp. 349-362, 2007.

[14] A. Famili, D. A. Ammar, and M. Y. Kahook, "Ethyl pyruvate treatment mitigates oxidative stress damage in cultured trabecular meshwork cells," Molecular Vision, vol. 19, pp. 13041309, 2013.

[15] K. K. Kao and M. P. Fink, "The biochemical basis for the antiinflammatory and cytoprotective actions of ethyl pyruvate and related compounds," Biochemical Pharmacology, vol. 80, no. 2, pp. 151-159, 2010.

[16] M. Shen, J. Lu, W. Dai et al., "Ethyl pyruvate ameliorates hepatic ischemia-reperfusion injury by inhibiting intrinsic pathway of apoptosis and autophagy," Mediators of Inflammation, vol. 2013, Article ID 461536, 12 pages, 2013.

[17] J. Guo, J. Zhang, X. Luo et al., "Effects of ethyl pyruvate on cardiac function recovery and apoptosis reduction after global cold ischemia and reperfusion," Experimental and Therapeutic Medicine, vol. 7, no. 5, pp. 1197-1202, 2014.

[18] J. B. Gross Jr., J. Reichen, T. B. Zeltner, and A. Zimmermann, "The evolution of changes in quantitative liver function tests in a rat model of biliary cirrhosis: correlation with morphometric measurement of hepatocyte mass," Hepatology, vol. 7, no. 3, pp. 457-463, 1987.

[19] Q. Yang, R. J. Xie, X. X. Geng, X. H. Luo, B. Han, and M. L. Cheng, "Effect of Danshao Huaxian capsule on expression of matrix metalloproteinase- 1 and tissue inhibitor of metalloproteinase-1 in fibrotic liver of rats," World Journal of Gastroenterology, vol. 11, no. 32, pp. 49534956, 2005.

[20] R. A. Olek, W. Ziolkowski, D. J. Flis et al., "The effect of ethyl pyruvate supplementation on rat fatty liver induced by a highfat diet," Journal of Nutritional Science and Vitaminology, vol. 59, no. 3, pp. 232-237, 2013.

[21] Y. Yu, Y. Yu, M. Liu et al., "Ethyl pyruvate attenuated coxsackievirus B3-induced acute viral myocarditis by suppression of HMGB1/RAGE/NF-KB pathway," SpringerPlus, vol. 5, no. 1, p. 215, 2016.

[22] M. E. Bianchi and A. A. Manfredi, "High-mobility group box 1 (HMGB1) protein at the crossroads between innate and adaptive immunity," Immunological Reviews, vol. 220, no. 1, pp. 35-46, 2007.

[23] M. Entezari, D. J. Weiss, R. Sitapara et al., "Inhibition of highmobility group box 1 protein (HMGB1) enhances bacterial clearance and protects against Pseudomonas aeruginosa pneumonia in cystic fibrosis," Molecular Medicine, vol. 18, no. 3, pp. 477-485, 2012.

[24] F. P. Wang, L. Li, J. Li, J. Y. Wang, L. Y. Wang, and W. Jiang, "High mobility group box-1 promotes the proliferation and migration of hepatic stellate cells via TLR4-dependent signal pathways of PI3K/Akt and JNK," PLoS ONE, vol. 8, no. 5, p. e64373, 2013.

[25] J. Lynch, S. Nolan, C. Slattery, R. Feighery, M. P. Ryan, and T. McMorrow, "High-mobility group box protein 1: a novel mediator of inflammatory-induced renal epithelialmesenchymal transition," American Journal of Nephrology, vol. 32, no. 6, pp. 590-602, 2010.

[26] H. S. Kim, I. H. Cho, J. E. Kim et al., "Ethyl pyruvate has an anti-inflammatory effect by inhibiting ROS-dependent STAT signaling in activated microglia," Free Radical Biology and Medicine, vol. 45, no. 7, pp. 950-963, 2008.

[27] S. W. Kim, H. K. Lee, J. H. Shin, and J. K. Lee, "Up-down regulation of HO-1 and iNOS gene expressions by ethyl pyruvate via recruiting p300 to Nrf2 and depriving it from p65," Free Radical Biology and Medicine, vol. 65, pp. 468-476, 2013.

[28] M. Mani, S. Khaghani, T. G. Mohammadi et al., "Activation of Nrf2-antioxidant response element mediated glutamate cysteine ligase expression in hepatoma cell line by homocysteine," Hepatitis Monthly, vol. 13, no. 5, p. e8394, 2013.

[29] J. S. Yu, W. C. Chen, C. K. Tseng et al., "Sulforaphane suppresses hepatitis $\mathrm{C}$ virus replication by up-regulating heme oxygenase-1 expression through PI3K/Nrf2 pathway," PLoS One, vol. 11, no. 3, p. e0152236, 2016.

[30] L. L. Qu, B. Yu, Z. Li, W. X. Jiang, J. D. Jiang, and W. J. Kong, "Gastrodin ameliorates oxidative stress and proinflammatory response in nonalcoholic fatty liver disease through the AMPK/Nrf2 pathway," Phytotherapy Research, vol. 30, no. 3, pp. $402-411,2016$. 
[31] M. Li, X. F. Wang, J. J. Shi et al., "Caffeic acid phenethyl ester inhibits liver fibrosis in rats," World Journal of Gastroenterology, vol. 21, no. 13, pp. 3893-3903, 2015.

[32] D. Gordillo-Bastidas, E. Oceguera-Contreras, A. SalazarMontes, J. Gonzalez-Cuevas, L. D. Hernandez-Ortega, and J. Armendariz-Borunda, "Nrf2 and Snail-1 in the prevention of experimental liver fibrosis by caffeine," World Journal of Gastroenterology, vol. 19, no. 47, pp. 9020-9033, 2013.

[33] J.-p. Li, Y. Gao, S.-f. Chu et al., "Nrf2 pathway activation contributes to anti-fibrosis effects of ginsenoside Rg1 in a rat model of alcohol- and CCl4-induced hepatic fibrosis," Acta Pharmacologica Sinica, vol. 35, no. 8, pp. 1031-1044, 2014.

[34] T. Satoh, T. Rezaie, M. Seki et al., "Dual neuroprotective pathways of a pro-electrophilic compound via HSF-1-activated heat-shock proteins and Nrf2-activated phase 2 antioxidant response enzymes," Journal of Neurochemistry, vol. 119, no. 3, pp. 569-578, 2011.

[35] S. Tanigawa, C. H. Lee, C. S. Lin et al., "Jun dimerization protein 2 is a critical component of the Nrf2/MafK complex regulating the response to ROS homeostasis," Cell Death \& Disease, vol. 4, p. e921, 2013.

[36] A. Parcellier, M. Brunet, E. Schmitt et al., "HSP27 favors ubiquitination and proteasomal degradation of p27Kipland helps S-phase re-entry in stressed cells," The FASEB Journal, vol. 20, no. 8, pp. 1179-1181, 2006. 


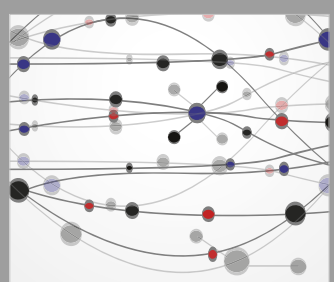

The Scientific World Journal
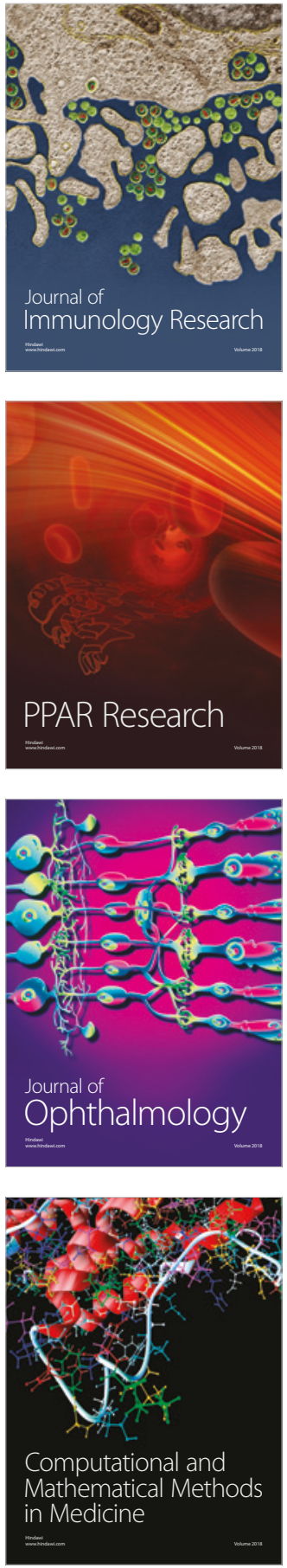

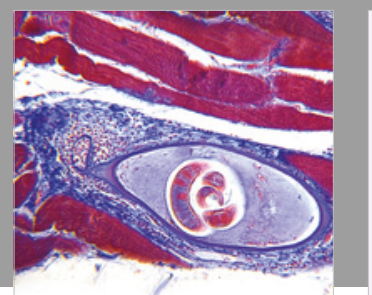

Gastroenterology Research and Practice

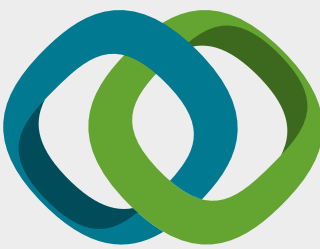

\section{Hindawi}

Submit your manuscripts at

www.hindawi.com
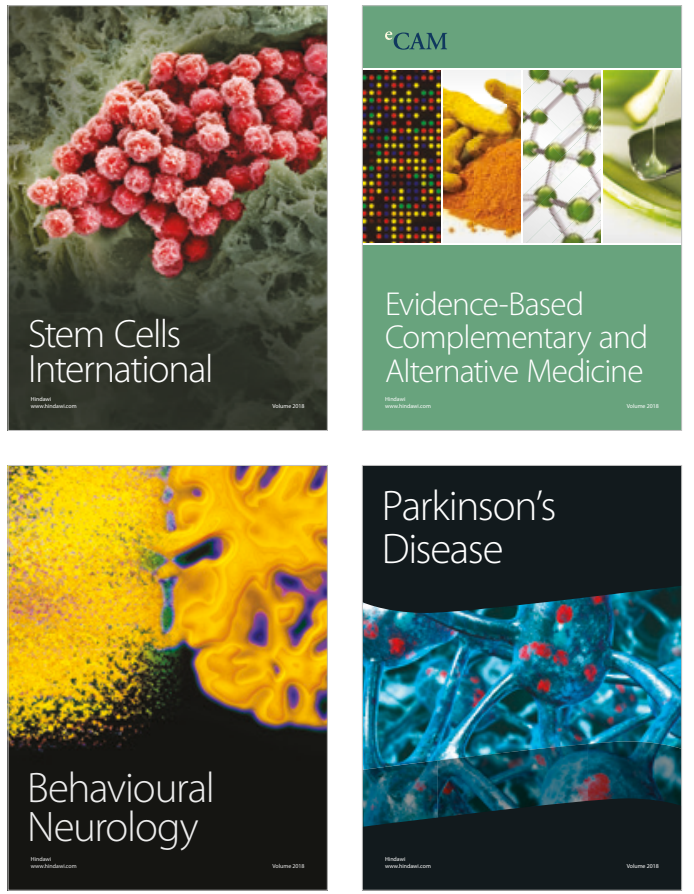

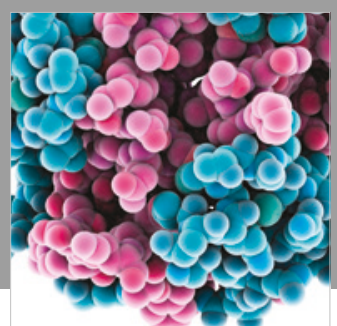

ournal of

Diabetes Research

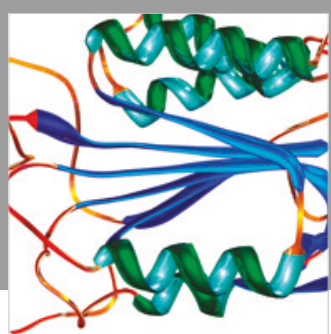

Disease Markers
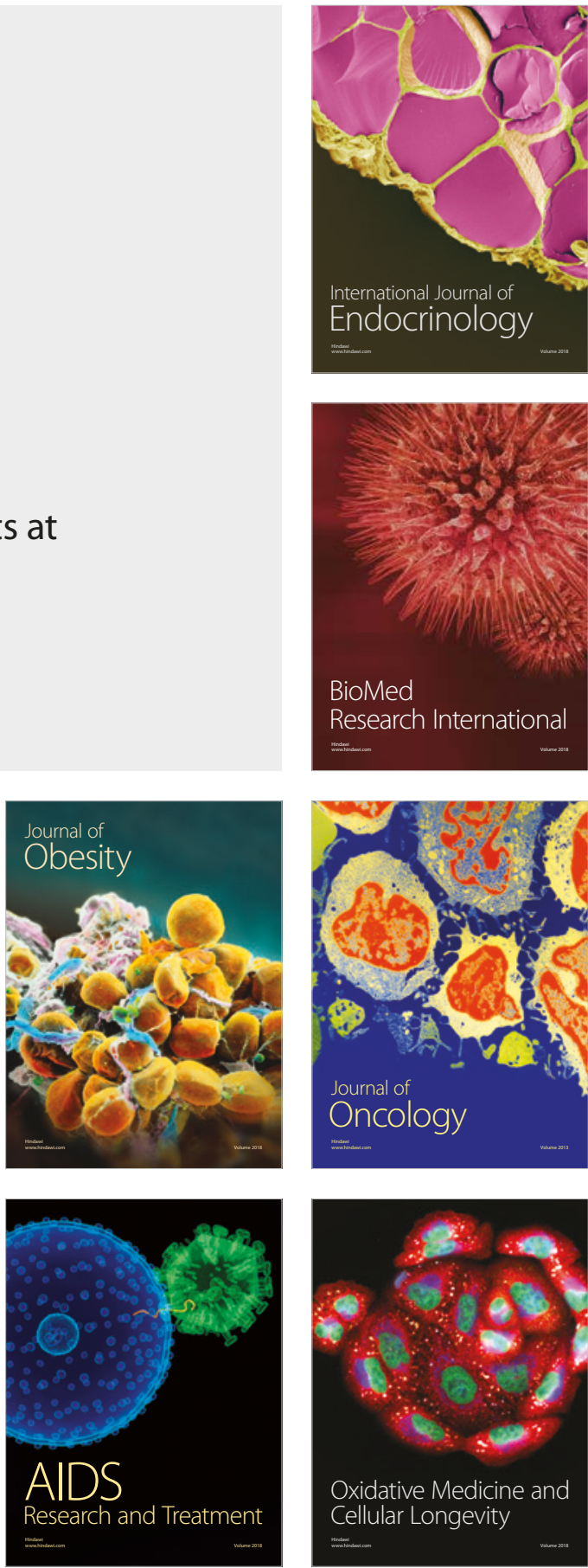